\title{
THE POINCARÉ INEQUALITY AND ENTIRE FUNCTIONS
}

\author{
J. MICHAEL PEARSON
}

(Communicated by J. Marshall Ash)

ABSTRACT. Inequalities for spaces of entire functions on $\mathbf{C}^{n}$, which generalize the Poincare inequality for Gaussian measure, are obtained. The relationship between these inequalities and hypercontractive estimates for diffusion semigroups are discussed.

\section{INTRODUCTION}

The Poincaré inequality for $\mathbf{R}^{n}$ is

$$
\int_{\mathbf{R}^{n}}|f|^{2} d \mu-\left(\int_{\mathbf{R}^{n}} f d \mu\right)^{2} \leq \int_{\mathbf{R}^{n}}|\nabla f|^{2} d \mu
$$

where $d \mu$ is normalized Gaussian measure on $\mathbf{R}^{n}$,

$$
d \mu=d \mu(x)=(2 \pi)^{-n / 2} e^{-|x|^{2} / 2} d x .
$$

In a recent paper, Beckner [Be2] discusses the role of this inequality in applications, especially in regards to quantum mechanics and the uncertainty principle, and obtains

Theorem 1 (Beckner). For $f \in L^{2}(d \mu), 1 \leq p \leq 2$, and $e^{-t}=\sqrt{p-1}$,

$$
\int_{\mathbf{R}^{n}}|f|^{2} d \mu-\int_{\mathbf{R}^{n}}\left|e^{-t N} f\right|^{2} d \mu \leq(2-p) \int_{\mathbf{R}^{n}}|\nabla f|^{2} d \mu
$$

and

$$
\int_{\mathbf{R}^{n}}|f|^{2} d \mu-\left(\int_{\mathbf{R}^{n}}|f|^{p} d \mu\right)^{2 / p} \leq(2-p) \int_{\mathbf{R}^{n}}|\nabla f|^{2} d \mu .
$$

Inequalities (1.2) and (1.3) can be viewed as interpolating inequalities for the Poincare inequality (1.1) and the logarithmic Sobolev inequality of Gross [G]

$$
\int_{\mathbf{R}^{n}}|f|^{2} \ln |f| d g-\int_{\mathbf{R}^{n}}|f|^{2} d g \ln \left(\int_{\mathbf{R}^{n}}|f|^{2} d g\right)^{1 / 2} \leq \int_{\mathbf{R}^{n}}|\nabla f|^{2} d g .
$$

Integrating by parts on the right-hand side of (1.1) yields

$$
\int_{\mathbf{R}^{n}}|f|^{2} d \mu-\left(\int_{\mathbf{R}^{n}} f d \mu\right)^{2} \leq \int_{\mathbf{R}^{n}}(N f) f d \mu,
$$

Received by the editors January 6, 1992.

1980 Mathematics Subject Classification (1985 Revision). Primary 42B99. 
where the number operator $N=-\Delta+x \cdot \nabla$ is the generator of the Hermite semigroup on $\mathbf{R}^{n}$, also called the Ornstein-Uhlenbeck process. Inequality (1.4) corresponds to Nelson's hypercontractive estimate for the Hermite semigroup $[\mathrm{N}]$ : for $1 \leq p \leq 2$,

$$
e^{-t} \leq \sqrt{p-1} \Rightarrow \int_{\mathbf{R}^{n}}\left|e^{-t N} f\right|^{2} d \mu \leq\left(\int_{\mathbf{R}^{n}}|f|^{p} d \mu\right)^{2 / p} .
$$

Beckner observed that inequality (1.3) follows from (1.2) by using (1.5) and that inequality (1.4) can be recovered from (1.3) by a limiting argument as $p$ approaches 2 .

In this note, we will obtain analogues of inequalities (1.2) and (1.3) for the spaces $A^{p}=A^{p}\left(\mathbf{C}^{n}\right), 0<p<\infty$, consisting of entire functions $\Phi$ on $\mathbf{C}^{n}$ such that

$$
\int_{\mathbf{C}^{n}}|\Phi(z)|^{p} d \mu<\infty
$$

where for $z=x+i y, x, y \in \mathbf{R}^{n}$,

$$
d \mu=d \mu(z)=(2 \pi)^{-n} e^{-|z|^{2} / 2} d|z|=d \mu(x) d \mu(y)
$$

denotes normalized Gaussian measure on $\mathbf{C}^{n}$. The Poincaré inequality in this setting is

$$
\int_{\mathbf{C}^{n}}|f|^{2} d \mu-(f(0))^{2} \leq 2 \int_{\mathbf{C}^{n}}\left|\frac{d f}{d z}\right|^{2} d \mu .
$$

In $\S 2$, we will prove

Theorem 2. For $f \in A^{2}\left(\mathbf{C}^{n}\right)$ and $|\omega| \leq 1$,

$$
\int_{\mathbf{C}^{n}}|f(z)|^{2} d \mu-\int_{\mathbf{C}^{n}}|f(\omega z)|^{2} d \mu \leq 2\left(1-|\omega|^{2}\right) \int_{\mathbf{C}^{n}}\left|\frac{d f}{d z}\right|^{2} d \mu,
$$

and, for $0<p \leq 2$,

$$
\int_{\mathbf{C}^{n}}|f(z)|^{2} d \mu-\left(\int_{\mathbf{C}^{n}}|f(z)|^{p} d \mu\right)^{2 / p} \leq(2-p) \int_{\mathbf{C}^{n}}\left|\frac{d f}{d z}\right|^{2} d \mu .
$$

Inequality (1.6) can be recovered from (1.7) by taking the limit as $|\omega| \rightarrow 0$.

Inequalities (1.7) and (1.8) interpolate between the Poincaré inequality (1.6) and the logarithmic inequality

$$
\int_{\mathbf{C}^{n}}|f|^{2} \ln |f| d \mu-\int_{\mathbf{C}^{n}}|f|^{2} d \mu \ln \left(\int_{\mathbf{C}^{n}}|f|^{2} d \mu\right)^{1 / 2} \leq \int_{\mathbf{C}^{n}}\left|\frac{d f}{d z}\right|^{2} d \mu .
$$

Inequality (1.9) corresponds to the following hypercontractive estimate: for $0<p \leq 2$,

$$
|\omega| \leq \sqrt{\frac{p}{2}} \Rightarrow \int_{\mathbf{C}^{n}}|f(\omega z)|^{2} d \mu \leq\left(\int_{\mathbf{C}^{n}}|f(z)|^{p} d \mu\right)^{2 / p} .
$$

Both (1.9) and (1.10) are due to Janson [J]. 
In this case, note that (1.8) follows from (1.7) by setting $\omega=\sqrt{p / 2}$ and using (1.10). We can then use a limiting argument as $p$ approaches 2 to recover inequality (1.9) from (1.8).

\section{Proof of Theorem 2}

From the preceding remarks, it will be enough to show that (1.7) holds. To obtain (1.7) we will need to recall some properties of $A^{2}$. The space $A^{2}$ arises in the study of canonical operators in quantum mechanics through the ideas of Fock, Dirac, Bargmann [Ba], and Segal [S1, S2]. The properties described below are worked out in detail in [Ba].

On $A^{2}$ the family of polynomials

$$
f_{\alpha}(z)=\frac{z^{\alpha}}{2^{(|\alpha|+n) / 4} \sqrt{\alpha !}}=\prod_{k=1}^{n} \frac{z_{k}^{\alpha_{k}}}{2^{\left(\alpha_{k}+1\right) / 4} \sqrt{\alpha_{k} !}},
$$

where $\alpha=\left(\alpha_{1}, \ldots, \alpha_{n}\right)$ is a multi-index of nonnegative integers, is an orthonormal basis with respect to the inner product

$$
\langle f, g\rangle=\int_{\mathbf{C}^{n}} \overline{f(z)} g(z) d \mu(z) .
$$

The operator (of multiplication by) $z_{k}$ is the adjoint of $2 \partial / \partial z_{k}=\partial / \partial x_{k}-$ $i \partial / \partial y_{k}$, and the analogue of the number operator in this setting is $N=z \cdot d / d z$. $N$ is a positive, selfadjoint operator, which acts on the basis according to the rule

$$
N f_{\alpha}=|\alpha| f
$$

One can also check that

$$
2 \int_{\mathbf{C}^{n}}\left|\frac{d f}{d z}\right|^{2} d \mu=\int_{\mathbf{C}^{n}} \overline{(N f)} f d \mu .
$$

It is easy to see that $N$ generates the semigroup $P(t)$, defined for $t \geq 0$ by

$$
(P(t) f)(z)=\left(e^{-t N} f\right)(z)=f\left(e^{-t} z\right)
$$

or, setting $e^{-t}=\omega$,

$$
(P(t) f)(z)=f(\omega z) .
$$

This makes the relationship between Theorems 1 and 2 clear. In fact, both inequalities (1.2) and (1.7) can be written in the form

$$
\int|f|^{2} d \mu-\int\left|e^{-t N} f\right|^{2} d \mu \leq\left(1-e^{-2 t}\right)\langle N f, f\rangle .
$$

The differences in Theorems 1 and 2 are primarily a result of the stronger hypercontractivity in the complex case and the fact that, since $d \mu(z)$ is invariant under complex rotations, we need not restrict $\omega$ to be real. We will now show inequality (1.7) to be a consequence of spectral considerations. To see this, let $\pi_{k}$ be the projection map from $A^{2}$ to the subspace spanned by the $f_{\alpha}$ 's with length $|\alpha|=k$. Then using (2.1) and (2.2), inequality (1.7) is equivalent to

$$
\int_{\mathbf{C}^{n}}\left|\pi_{k} f\right|^{2} d \mu-|\omega|^{2 k} \int_{\mathbf{C}^{n}}\left|\pi_{k} f\right|^{2} d \mu \leq\left(1-|\omega|^{2}\right) k \int_{\mathbf{C}^{n}}\left|\pi_{k} f\right|^{2} d \mu .
$$


Inequality $(2.3)$ is determined by the relation

$$
1-|\omega|^{2 k} \leq\left(1-|\omega|^{2}\right) k,
$$

which is a consequence of the identity

$$
1-|\omega|^{2 k}=\left(1-|\omega|^{2}\right)\left(1+|\omega|^{2}+|\omega|^{4}+\cdots+|\omega|^{2(k-1)}\right)
$$

and the fact that, with $|\omega| \leq 1,\left(1+|\omega|^{2}+|\omega|^{4}+\cdots+|\omega|^{2(k-1)}\right) \leq k$.

Since $|\omega| \leq 1$ and $k \geq 1$ implies $1 \leq\left(1-|\omega|^{2 k}\right) /\left(1-|\omega|^{2}\right)$, inequality (1.7) gives the following smooth interpolation for the Poincare inequality (1.6):

$$
\begin{aligned}
\int_{\mathbf{C}^{n}}|f(z)|^{2} d \mu-(f(0))^{2} & \leq\left(\frac{\int_{\mathbf{C}^{n}}|f(z)|^{2} d \mu-\int_{\mathbf{C}^{n}}|f(\omega z)|^{2} d \mu}{1-|\omega|^{2}}\right) \\
& \leq 2 \int_{\mathbf{C}^{n}}\left|\frac{d f}{d z}\right|^{2} d \mu .
\end{aligned}
$$

\section{FURTHER RESULTS}

Results similar to Theorems 1 and 2 can be obtained for the sphere $S^{n}$ by using the technique of $\S 2$ and either the hypercontractive estimates for the heat semigroup due to Mueller and Weissler [MW] or those for the Poisson semigroup due to Beckner [Be1]. In fact, this was carried out for the Poisson semigroup by Beckner [Be2], who found that, for $1 \leq p \leq 2$,

$$
\int_{S^{n}}|F|^{2} d \xi-\left(\int_{S^{n}}|F|^{p} d \xi\right)^{2 / p} \leq \frac{2-p}{n} \int_{S^{n}}|\nabla F|^{2} d \xi,
$$

where $d \xi$ denotes normalized surface measure on $S^{n}$.

Let $P^{2}$ denote 2-dimensional real projective space, which we view as $S^{2}$ with antipodal points identified. We again use $d \xi$ to denote normalized surface measure. Then using (3.1) and the method of proof in the author's paper [P], we obtain

Theorem 3. For $F \in L^{2}\left(P^{2}\right)$ and $1 \leq p \leq 2$,

$$
\int_{P^{2}}|F|^{2} d \xi-\left(\int_{P^{2}}|F|^{p} d \xi\right)^{2 / p} \leq \frac{2-p}{4} \int_{P^{2}}|\nabla F|^{2} d \xi .
$$

If we divide both sides of $(3.2)$ by $(2-p)$ and take the limit as $p$ approaches 2 , we get the logarithmic inequality

$$
\int_{P^{2}}|F|^{2} \ln |F| d \xi-\left(\int_{P^{2}}|F|^{2} d \xi\right) \ln \left(\int_{P^{2}}|F|^{2} d \xi\right)^{1 / 2} \leq \frac{1}{4} \int_{P^{2}}|\nabla F|^{2} d \xi
$$

Inequality (3.3) corresponds to the hypercontractive estimate for the heat semigroup on $P^{2}$ : for $1 \leq p \leq 2$,

$$
e^{-4 t} \leq \sqrt{p-1} \Rightarrow \int_{P^{2}}\left|e^{t \Delta} F\right|^{2} d \xi \leq\left(\int_{P^{2}}|F|^{p} d \xi\right)^{2 / p} .
$$

Both (3.3) and (3.4) appear in [P]. 


\section{REFERENCES}

[Ba] V. Bargmann, On a Hilbert space of analytic functions and an associated integral transform, Part I, Comm. Pure Appl. Math. 14 (1961), 187-214.

[Be1] W. Beckner, Sobolev inequalities, the Poisson semigroup and analysis on the sphere $S^{n}$, Proc. Nat. Acad. Sci. U.S.A. 89 (1992), 4816-4819.

[Be2] __ A generalized Poincaré inequality for Gaussian measures, Proc. Amer. Math. Soc. 105 (1989), 397-400.

[G] L. Gross, Logarithmic Sobolev inequalities, Amer. J. Math. 97 (1975), 1061-1083.

[J] S. Janson, On hypercontractivity for multipliers on orthogonal polynomials, Ark. Mat. 21 (1983), 97-110.

[MW] C. E. Mueller and F. B. Weissler, Hypercontractivity for the heat semigroup for ultraspherical polynomials and on the n-sphere, J. Funct. Anal. 48 (1982), 252-283.

[N] E. Nelson, The free Markov field, J. Funct. Anal. 12 (1973), 211-227.

[P] J. M. Pearson, A hypercontractive estimate for the heat semigroup on $P^{2}$, Michigan Math. J. 37 (1990), 439-446.

[S1] I. E. Segal, Foundations of the theory of dynamical systems of infinitely many degrees of freedom. I, Mat.-Fys. Medd. Danske Vid. Selsk. 31 (1959).

[S2] _ Mathematical problems of relativistic physics, Amer. Math. Soc., Providence, RI, 1963.

Department of Mathematics, Florida International University, Miami, Florida 33199 Current address: Department of Mathematics and Statistics, Mississippi State University, Mississippi State, Mississippi 39762

E-mail address: pearson@math.msstate.edu 